\title{
Differential Expression of Serum Ceruloplasmin And a2-HS Glycoprotein among Nasopharyngeal Carcinoma Patients
}

Saied Reza Doustjalali ${ }^{1 *}$, Munira Bhuiyan ${ }^{1}$, Karim Al-Jashamy ${ }^{1}$, Mohammed Irfan ${ }^{1}$, Magdi El Sersi ${ }^{1}$, Khin Thant Zin ${ }^{1}$, Nyan Htain Linn ${ }^{1}$, Wai Ma Lin ${ }^{1}$, Vinothini Appalanaidu ${ }^{1}$, Samiah Yasmin Abdul Kadir ${ }^{1}$, Jeyaseelan Nadankutty ${ }^{1}$, Rohaini Mohamad ${ }^{1}$, Viswanathan Neelakantan ${ }^{1}$, Hafiza Arzuman ${ }^{1}$, Wong A-Chin ${ }^{1}$, Htet Htet $^{1}$, Ahmad Yusuf ${ }^{1}$, Harmiza Harun ${ }^{1}$, Ali Yaldrum ${ }^{2}$, Aida Nur Ashikin Abdul Rahman ${ }^{3}$, Marzalina Mansor ${ }^{4}$, Shamala Devi Sekaran ${ }^{5}$ and Negar Shafiei Sabet $^{1,5}$

${ }^{1}$ Faculty of Medicine, SEGi University, Kota Damansara, Selangor, Malaysia

${ }^{2}$ Faculty of Dentistry, SEGi University, Kota Damansara, Selangor, Malaysia

${ }^{3}$ Faculty of Dentistry, Universiti Teknologi Mara (UiTM), Shah Alam, Selangor, Malaysia

${ }^{4}$ Forest Research Institute Malaysia (FRIM), Selangor, Malaysia

${ }^{5}$ Tropical Infectious Diseases Research and Education Center (TIDREC), Department of Medical Microbiology, Faculty of Medicine, University of Malaya, Kuala Lumpur, Malaysia

"Corresponding authors: Saeid Reza Doustjalali and Negar Shafiei Sabet, Faculty of Medicine, SEGi University, Malaysia, Tel: +60 3-6145 1777; E-mail: saeidrezad@yahoo.com, negar_4@yahoo.com

Rec Date: Apr 02, 2015; Acc Date: May 25, 2015; Pub Date: May 30, 2015

Copyright: (c) 2015, Doustjalali SR, et al. This is an open-access article distributed under the terms of the Creative Commons Attribution License, which permits unrestricted use, distribution, and reproduction in any medium, provided the original author and source are credited.

\begin{abstract}
The two-dimensional gel electrophoresis (2-DE) approach was used to evaluate the simultaneous expression of serum proteins in patients with nasopharyngeal carcinoma (NPC) and to detect differentially expressed proteins which could be used as specific and sensitive biomarkers for early diagnosis of the disease. We have subjected unfractionated whole sera of ten newly diagnosed Malaysian Chinese patients with World Health Organization (WHO) type III NPC to 2-DE and image analysis. The results obtained were then compared to that generated from sera of ten normal healthy controls of the same ethnic group and range of age. Our data showed higher expression of ceruloplasmin (CPL) and lower expression of a2-HS glycoprotein (AHS) in the serum high abundance 2-DE protein profiles of NPC patients as compared to that of the normal healthy controls. The ceruloplasmin (CPL) and a2-HS glycoprotein (AHS) spots were identified by mass spectrometric analysis and Mascot database search. In conclusions, this information may help in early diagnosis of nasopharyngeal carcinoma.
\end{abstract}

Keywords: Ceruloplasmin; $\alpha 2-\mathrm{HS}$ glycoprotein; Nasopharyngeal carcinoma; Serum 2-DE proteomics

\section{Abbreviations:}

AAT: a1-antitrypsin; ABG: a1-B glycoprotein; AHS: a2-HS glycoprotein; CFB: Complement factor B; CLU: Clusterin; CPL: Ceruloplasmin; HAP: Haptoglobin ( $\beta$ chain); PR1 to 3: unidentified proteins

\section{Introduction}

Nasopharyngeal carcinoma (NPC) is the cancer occurring in the mucosal lining (squamous, columnar, and transitional epithelium) and the minor salivary glands of nasopharynx [1]. It is a rare malignancy in western countries (less than 1 case per 100,000 person-years), and it is one of the most confusing, commonly misdiagnosed and poorly understood disease. But it occurs more frequently in China (more than 20 cases per 100,000 person-years among Southern Chinese) and Southeast Asia [2]. The rate of incidence generally increases from ages 20 years to around 50 years [3]. In Malaysia, NPC is the third most common cancer in men (male: 8.5 cases per 100,000 person-years and female: 2.6 cases per 100,000 person-years) and the rate is highest among the Chinese men (15.9 cases per 100,000 person-years) [4].

According to the World Health Organization (WHO), nasopharyngeal carcinoma is classified into three histological categories. Type I represents well to moderately differentiated squamous cell carcinomas with keratin production. Type II includes nonkeratinizing carcinomas. Type III comprises a diverse group of carcinomas, and these lesions often are described as undifferentiated carcinomas or lymphoepitheliomas. Types II and III are commonly associated with elevated Epstein-Barr virus (EBV) titers and have a better prognosis than type I [2].

Nasopharyngeal carcinoma shows a remarkably high cure rate for early-stage of disease, and early detection is critical to improve the overall prognosis of patients. However, the presenting clinical features of NPC are often nonspecific, and examination of nasopharynx requires expertise and renders early detection is difficult. Thus, diagnosis of NPC is mainly made by biopsy of the nasopharyngeal mass [5,6]. At present, the detection of IgA (Immunoglobulin A) antibodies to EBV specific antigens is the most common serological aid to the diagnosis of NPC [7].

The proteomics approach offer paradigm shift in studies on the simultaneous expression of serum proteins in patients with cancer. Detection of selective or aberrantly expressed serum proteins in cancer patients may prompt investigations of their potential application as novel diagnostic or prognostic biomarkers. Few biomarkers have been identified and proposed by proteomics technologies in NPC, and include: ceruloplasmin, Fibronectin, Mac-2 binding protein, Plasminogen activator inhibitor 1, and Inter-alpha-trypsin inhibitor precursor $[3,6,8]$. Some metabolites also appear promising to be useful biomarkers for diagnosis of NPC, and they are 
hydroxyphenylpyruvate, N-acetylglucosaminylamine, acetylglucosamine, and kynurenine [6].

In the present study, it was thought worthwhile to use the application of two-dimensional gel electrophoresis (2-DE) in serum of Malaysian NPC patients to detect differentially expressed proteins which could be used as specific and sensitive biomarkers for early diagnosis of the disease. Hence, we have subjected unfractionated whole sera of ten Malaysian Chinese patients with NPC and ten normal healthy controls of the same ethnic group and range of age for 2 -DE and image analysis. The differentially expressed proteins were identified by mass spectrometric analysis and Mascot database search.

\section{Materials and Methods}

\section{Serum samples}

Ten serum samples were gathered from 7 males and 3 females of Malaysian Chinese patients with NPC, ages ranged within 40-65 years old. These were used as routine laboratory testing prior to the treatment. Through histopathological tests, all patients were confirmed having undifferentiated carcinoma or WHO Type III NPC for either stage T1N1M0 or stage T2N2M0 [2]. Meanwhile the control sera were obtained from another ten normal healthy Malaysian Chinese volunteers, within the gender distribution and range of age as the patients. All sera samples were kept at $-20^{\circ} \mathrm{C}$ and subjected to similar treatment. This research was conducted in the Proteomic and Drug Discovery laboratory in Forest Research Institute of Malaysia (FRIM). The research proposal was approved by the Ethics Committee of SEGi University.

\section{Two-dimensional gel electrophoresis}

2-DE was performed as described before by using pre-casting immobilized dry strips of Amersham Biosciences, Uppsala, Sweden [8-11]. Whereby seven $\mu \mathrm{l}$ (450 $\mu \mathrm{g}$ protein) of unfractionated whole human serum samples were placed onto $11 \mathrm{~cm}$ isoelectric rehydrated pre- casting immobilized dry strips having $\mathrm{pH}$ between 4 to 7 . Meanwhile for the second dimension analysis, these strips were then subjected to electrophoresis using $8 \%$ to $18 \%$ gradient polyacrylamide gel in the presence of sodium dodecyl sulphate. Three replicates of tests were conducted and analyzed.

\section{Silver staining}

The 2-DE gels were developed by silver staining as previously described by Heukeshoven and Dernick [12]. For mass spectrometric analysis, gels were stained according to the method of Shevchenko [13].

\section{MALDI-ToF pro analysis}

All resolved proteins spots appeared in protein profiles were identified using the standard plasma protein reference, SWISS ExPASy [14]. The appearance of a1-antitrypsin (AAT), a1-B glycoprotein (ABG), a2-HS glycoprotein (AHS), complement factor B (CFB), clusterin (CLU) and the $\beta$ chain of haptoglobin (HAP), were confirmed as described before [9]. The identification of ceruloplasmin (CPL) and a2-HS glycoprotein (AHS) spots were carried out similarly by using the Ettan MALDI-ToF Pro, while gel trypsin digestion was performed according to the method of Shevchenko [13]. Mass analyses were conducted using a mixture $1 \mu \mathrm{l}$ of extracted sample with equal volume of matrix solution consisting of $10 \mathrm{mg} / \mathrm{ml}$ alpha-cyano-4hydroxycinnamic acid in $0.5 \%$ trifluoroacetic acid (TFA) and $50 \%$ acetonitrite (ACN). Only an amount of $0.3 \mu \mathrm{l}$ of the solution was placed onto the slide loader.

\section{Database search}

This study utilized Mascot program (www.matrixscience.com) to search for protein database. This program makes use of the peptide mass fingerprints (PMFs) to locate for database of matching peptides from known proteins. The following keywords were used during the search: trypsin digests (one missed cleavage allowed), species: Homo sapiens, mass value: monoisotropic, peptide mass tolerance: $\pm 0.1 \mathrm{Da}$, peptide charges state: $1+$ and NCBInr database. Protein identification was further subject to Amersham Biosciences Ettan MALDI software for confirmation.

\section{Image analysis}

Using Molecular Analyst PD Quest densitometry software (BioRad, Hercules, Calif., USA), protein spots were analyzed in terms of volume. The background noise was subtracted out and the analysis was confined to ten clusters of protein spots with $M_{r} \geq 30,000$, distinctively separated by $2-\mathrm{DE}$, having AAT, ABG, AHS, CFB, CLU, CPL, HAP and three unidentified proteins termed PR1, PR2 and PR3. While majority of non-dissolve proteins such as albumin, serum polypeptides having idiotypic and/or allotypic variations (the heavy and light chains of all isotypes of immunoglobulin and the a chains of haptoglobin) and those having low $\mathrm{M}_{\mathrm{r}}$ protein spots were not assessed. In this study, the percentage of volume contribution refers to the volume percentage of a protein taken against the total spot volume of all proteins including the unresolved peptides in each gel.

\section{Statistical analysis}

The values were presented as mean with \pm SD (standard deviation). This study used independent samples $t$ test to analyze the significance of differences between normal subjects and patients. A $p$ value of less than 0.05 was considered as having significant different.

\section{Results and Discussion}

Our procedure to separate the unfractionated whole sera of normal healthy individuals by 2 -DE created typical high resolution serum profiles consisting only the high abundance proteins. Based on the conditions of our experiments, protein spots that are usually generated and observed by silver staining were albumin, the heavy and light chains of IgA, IgG, IgM, a1- antitrypsin, a1-B glycoprotein, a2-HS glycoprotein, complement factor $B$, clusterin, $\beta$ chain of haptoglobin and three other unidentified protein spot clusters (Figure 1a). The identities of the above known high abundance acute-phase proteins have been reported before [9]. Similarly when we subjected unfractionated serum samples from 7 males and 3 females of newly diagnosed patients with NPC to 2-DE and silver staining based on the same experimental conditions, comparable results were obtained, with the exception of differentially expressed proteins CPL and AHS. Figure $1 \mathrm{~b}$ demonstrates a typical representative unfractionated serum protein profile of patients with NPC.

In our current study, image analysis performed on the silver-stained 2-DE serum protein profiles of ten NPC patients as compared to ten 
normal controls indicated significantly higher expression of CPL $(\mathrm{p}=0.0001)$ and lower expression of AHS $(\mathrm{p}=0.01)$ in patients.
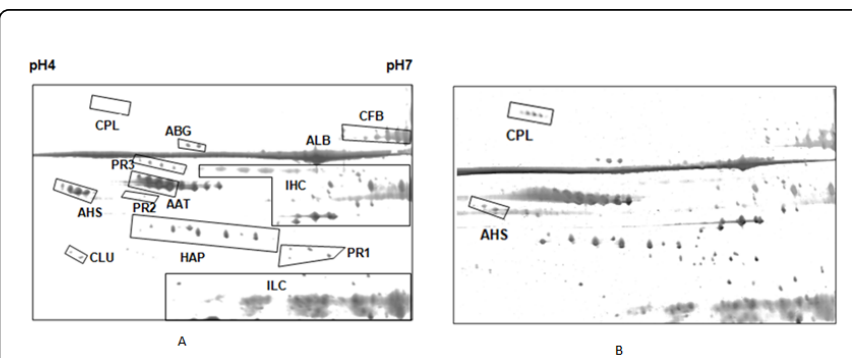

Figure 1: Typical 2-DE serum protein profiles of normal healthy adult controls and patients with NPC. We subjected serum samples of normal healthy controls (a) patients with NPC prior to treatment (b) to 2-DE (pI 4-7) and silver staining. In addition to albumin (ALB), immunoglobulin $\alpha, \gamma$ and $\mu$ heavy chains (IHC) and immunoglobulin light chains (ILC), other high abundance protein spots were detected, such as al-antitrypsin (AAT), a1-B glycoprotein (ABG), a2-HS glycoprotein (AHS), complement factor $\mathrm{B}$ (CFB), clusterin (CLU), the $\beta$ chain of haptoglobin (HAP) and three unidentified proteins termed PR1, PR2 and PR3. Comparable expression was seen for most of the resolved proteins with exception of higher expression of CPL and lower expression of AHS in NPC patients. Acid sides of all 2-DE gels are to the left and relative molecular mass declines from the top.

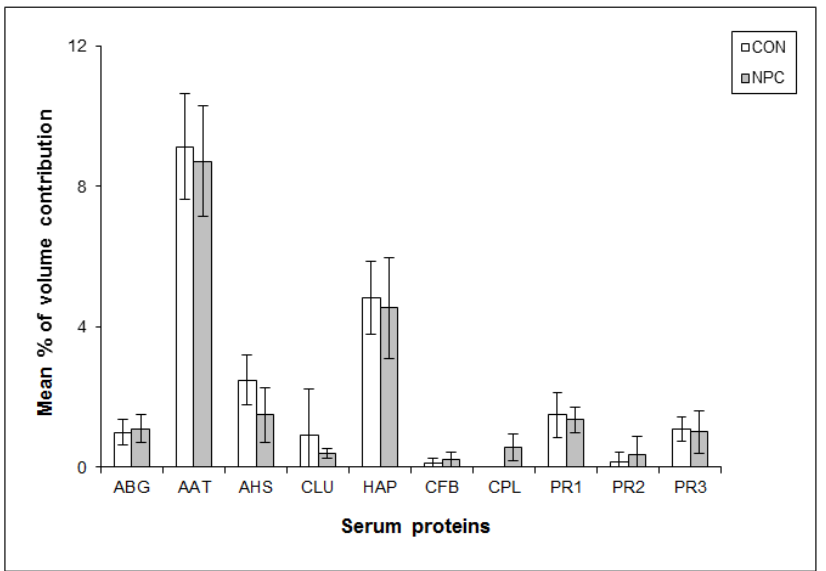

Figure 2: Mean percentage of volume contribution of 2-DE detectable serum proteins of patients with NPC. The Molecular analyst PD Quest densitometry software (Bio-Rad, Hercules, Calif., USA) was used to analyze the volumes of protein spots. AAT: a1antitrypsin, ABG: a1-B glycoprotein, AHS: a2-HS glycoprotein, CFB: complement factor B, CLU: clusterin, CPL: ceruloplasmin, HAP: haptoglobin ( $\beta$ chain), PR1 to 3 : unidentified proteins. The normal controls and newly diagnosed patients with nasopharyngeal carcinoma prior to treatment were shown as CON and NPC respectively. Asterisk denotes the value of statistical significant difference.

However, comparable results were obtained for all the other high abundance serum proteins analyzed (Figure 2). Both CPL (Figure 3) and AHS (Figure 4) spots were identified by using the Ettan MALDIToF Pro mass spectrometry via in gel trypsin digestion. The results of this study showed that 2-DE is highly reproducible in the NPC. However, one previous report has indicated that image analysis performed on triplicate 2-DE gels of serum samples produced minimal relative standard deviation in percentage values of volume contribution of all serum protein spots analyzed [9]. Another previous study has also shown the over expression and highest serum levels of CPL in nasopharyngeal cancer patients [8]. The higher levels of ceruloplasmin (CPL) in sera of patients with various types of cancers have also been previously described [15-23].
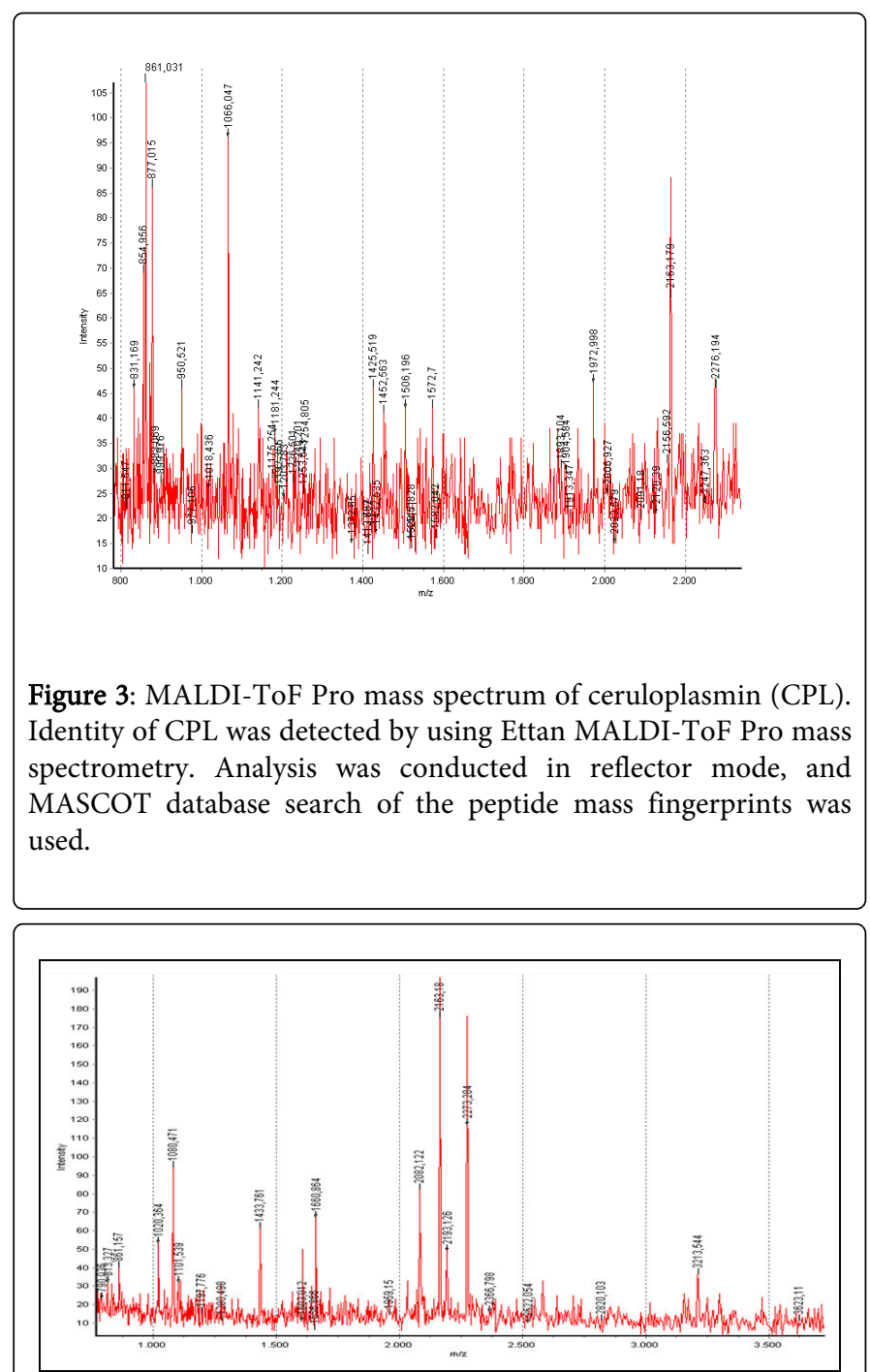

Figure 4: MALDI-ToF Pro mass spectrum of a2-HS glycoprotein (AHS). Identity of ASH was detected by using Ettan MALDI-ToF Pro mass spectrometry. Analysis was conducted in reflectors mode, and MASCOT database search of the peptide mass fingerprints was used.

In the present study, the higher expression of CPL was again detected in the sera of all NPC patients. In addition to CPL, the expression of AHS was lower in sera of patients while most of the other high abundance serum proteins resolved by 2-DE was 
comparable to that of the control sera. Lower serum level of AHS in nasopharyngeal cancer was previously reported [24] which supports our finding in this study. The CPL and AHS spots were identified by mass spectrometric analysis and Mascot database search. The sera of NPC patients generated typical protein profiles that were also different from the earlier reported 2-DE protein profiles of patients with breast cancer and fibrocystic disease of the breast [9]. Our data showed higher expression of CPL and lower expression of AHS in serum high abundance 2-DE protein profiles of NPC patients as compared to that of the normal healthy controls. In conclusion, this information may help in early diagnosis of nasopharyngeal carcinoma.

\section{Acknowledgement}

This study was supported by Ministry of Higher Education (MOHE), Malaysia under the High Impact Research (HIR)-MOHE UM.C/HIR/MOHE/MED/40, 848 20001-E000079 and Fundamental Research Grant Scheme (FRGS) FP027-2010B.

\section{References}

1. Wei KR, Xu Y, Liu J, Zhang WJ, Liang ZH (2011) Histopathological classification of Nasopharyngeal carcinoma. Asian Pac J Cancer Prev 12: 1141-1147.

2. Sun LM, Epplein M, Li CI, Vaughan TL, Weiss NS (2005) Trends in the incidence rates of nasopharyngeal carcinoma among Chinese Americans living in Los Angeles County and the San Francisco Metropolitan area, 1992-2002. Am J Epidemiol 162: 1174-1178.

3. Cho WC (2007) Nasopharyngeal carcinoma: molecular biomarker discovery and progress. Mol Cancer. 6:1.

4. http://www.makna.org.my/PDF.

5. Schantz SP, Harrison LB, Forastiere AA (1997) Tumors of the nasal cavity and paranasal sinuses, nasopharynx, oral cavity and oropharynx, Cancer: Principles and Practice of Oncology. (5thedn), Lippincott- Raven Publishers, Philadelphia, USA.

6. Tang F, Xie C, Huang D, Wu Y, Zeng M, et al. (2011) Novel potential markers of nasopharyngeal carcinoma for diagnosis and therapy. Clin Biochem 44: 711-718.

7. Tsang RK, Vlantis AC, Ho RW, Tam JS, To KF, et al. (2004) Sensitivity and specificity of Epstein-Barr virus IgA titer in the diagnosis of nasopharyngeal carcinoma: a three-year institutional review. Head Neck 26: 598-602.

8. Doustjalali SR, Yusof R, Govindasamy GK, Bustam AZ, Pillay B, et al. (2006) Patients with nasopharyngeal carcinoma demonstrate enhanced serum and tissue ceruloplasmin expression. J Med Invest 53: 20-28.

9. Doustjalali SR, Yusof R, Yip CH, Looi M, Pillay B, et al. (2004) Aberrant expression of acute-phase reactant proteins in sera and breast lesions of patients with malignant and benign breast tumors. Electrophoresis 25 : 2392-2401.
10. Min HN, Saeid R, Rajiv P, Negar SS, Sok F, et al. (2013) Two dimensional protein map standardization of human bone marrow stromal cells. J Proteomics Bioinformatics 6: 85-88.

11. Doustjalali SR, Munira B, Karim Al-Jashamy, Nyan HL, Samiah Y, et al. (2014) Two dimensional gel electrophoresis: An overview of proteomic technique in cancer research. Journal of Proteomics and Bioinformtics 7: 3077-3081.

12. Heukeshoven J, Dernick R (1988) Improved silver staining procedure for fast staining in Phast System development unit. I. Staining of sodium dodecyl sulphate gels. Electrophoresis 9: 28-32.

13. Shevchenko A, Wilm M, Vorm O, Mann M (1996) Mass spectrometric sequencing of proteins from silver-stained polyacrylamide gels. Anal Chem 68: 850-858.

14. Sanchez JC, Appel RD, Golaz O, Pasquali C, Ravier F, et al. (1995) Inside SWISS-2DPAGE database. Electrophoresis 16: 1131-51.

15. Pejovic M, Djordjevic V, Ignjatovic I, Stamenic T, Stefanovic V (1997) Serum levels of some acute phase proteins in kidney and urinary tract urothelial cancers. Int Urol Nephrol 29: 427- 432.

16. Agroyannis B, Dalamangas A, Dardouphas K, Fortoynas C, Saloum G, et al. (1994) Serum transferrin and ceruloplasmin in patients with cancer of the gastrointestinal and other systems. Anticancer Res 14: 2201-2203.

17. Arumanayagam M, Wong FW, Rogers M, Swaminathan R (1993) Serum ceruloplasmin, plasma copper concentration and copper to ceruloplasmin ratio in cervical carcinoma. Gynecol Obstet Invest 35: 175-178.

18. Kirchheimer JC, Kolbi H, Tatra G, Binder BR (1990) Relationship between plasma levels of components of the fibrinolytic system and acute-phase reactants in patients with uterine malignancies. Eur J Clin Invest 20: 79-84

19. Ros-Bullon MR, Sanchez-Pedreno P, Martinez-Liarte JH (2001) Serum ceruloplasmin in melanoma patients. Anticancer Res 21: 629-632.

20. Senra VA, Lopez SJJ, Quintela SD (1997) Serum Ceruloplasmin as a diagnostic marker of cancer. Cancer Lett 121: 139-145.

21. Nayak SB, Bhat VR, Upadhyay D, Udupa SL (2003) Copper and Ceruloplasmin status inserum of prostate and colon cancer patients. Indian J Physiol Pharmacol 47: 108-110.

22. Kasprzyk M, Dyszkiewicz W, Zwaruń D, Leśniewska K, Wiktorowicz K (2008) The assessment of acute phase proteins as prognostic factors in patients surgically treated for non-small cell lung cancer. Pneumonol Alergol Pol 76: 321-326.

23. Jayadeep A, Pillai KR, Kannan S, Nalinakumari KR, Mathew B, et al. (1997) Serum levels of copper, zinc, iron and ceruplasmin in oral leukoplakia and squamous cell carcinoma. J Exp Clin Cancer Res 16: 295-300.

24. Amold MB, Paul B, Yang C, Gregory T, Robert MW, et al. (1979) Serum glycoproteins and immunoglobulins in nasopharyngeal carcinoma: correlations with Epstein-Barr virus associated antibodies and clinical tumor stage. The American Journal of Surgery 138: 478-488. 